\title{
Le temps d'être humain...
}

\section{Yves Coppens}

Y. Coppens: membre de l'académie des sciences et de l'académie nationale de médecine, professeur au collège de France. Collège de France, 11, place Marcellin-Berthelot, 75231 Paris Cedex 05, France.

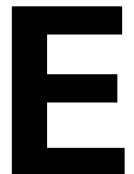

$\mathrm{n}$ introduction à ce très beau numéro de médecine/ sciences sur le temps en biologie, je suis évidemment tenté de dépasser tout de suite le temps de l'individu tel qu'il est à juste raison traité dans ces pages, pour rejoindre celui, paléobiologique, qui est ma dimension habituelle de travail.

La vie a plus de 4 milliards d'années et certaines des Archéobactéries de milieux particuliers, tels les croûtes de carbonate de sodium de certains lacs tropicaux, pourraient bien représenter aujourd'hui les descendantes peu transformées de ces formes unicellulaires sans noyau des commencements. Mais cette longévité est exceptionnelle. Le merveilleux arbre phylétique des êtres vivants est fait de plus de branches interrompues que de rameaux encore en fleurs et l'histoire de la vie, par ailleurs étonnamment inventive, est ainsi jalonnée d'événements cosmiques, géologiques, géographiques et consécutivement climatiques, environnementaux, écologiques qui en ont quelque peu bousculé le cours. Il n'en demeure pas moins vrai que, de même que l'individu en fonction de l'espèce à laquelle il appartient, a une certaine durée d'existence, l'espèce elle même, en fonction de l'ordre auquel son genre est rattaché et, semble-t-il, en fonction de sa taille aussi, a un certain temps de vie; ces temps sont certes très variables d'une extrémité du monde vivant à l'autre mais on parvient cependant à estimer certaines de ces durées; elles seraient de 500000 ans à quelques millions d'années par exemple pour les grands mammifères.

Il n'est pas inintéressant de rappeler que l'Homo sapiens, qui est une espèce de grand mammifère, a déjà consommé près d'un demi-million d'années; on peut heureusement faire valoir le fait qu'en ce qui le concerne, sapiens, comme erectus et ergaster voire habilis et rudolfensis qui le précèdent, n'est peut-être pas une véritable espèce biologique, mais un des grades, le dernier pour le moment, $d$ 'une seule et même vieille espèce humaine, espèce sur laquelle a rétroagi le milieu nouveau dit culturel que depuis 3 millions d'années elle a inventé. Cette dimension culturelle s'est d'ailleurs développée depuis à un point tel que l'homme moderne est désormais en train d'acquérir la maîtrise de son génome et qui sait, peut être celle de sa propre évolution biologique; il recherche en outre, avec un immense espoir de jeunesse éternelle, le gène du vieillissement de l'individu et la manière la plus habile d'en avoir le contrôle.

Chacun sait que cet homme moderne, gourmand de tout profit, a déjà gagné bien des années. C'est la première fois dans une espèce humaine que I'on parle dans certaines populations d'espérance de vie de plus de 70 ans. Les petits Lémuriens des zoos, forcément assistés dans la recherche de leur alimentation qu'ils ne peuvent se procurer eux-mêmes, gagnent comme les humains des années d'existence mais aussi et de manière significative, diverses modifications physiques et biologiques, des pathologies dégénératives par exemple, et même des cataractes et des dépigmentations du pelage! II est quand même amusant de constater le caractère fini de tout le contenu cosmique - toutes les étoiles s'éteignent - et biologique - la mort fait partie de la vie - d'un contenant, infini au moins dans sa réputation.

La véritable perception des milliards d'années du temps cosmique, géologique et paléobiologique et son éta- 
lonnage ne datent en fait que d'un demi-siècle. Et ce temps-là, et son extravagante épaisseur, représentent certainement un des grands acquis scientifiques du $X x^{e}$ siècle, avec l'électronique et le nucléaire; c'est d'ailleurs pour un certain nombre de ces méthodes de datation, du nucléaire. Et du même coup, alors que la Sorbonne, à la fin des années 1950, n'osait pas encore parler d'un million d'années pour l'homme, le public scientifique et tous les publics de la Terre se sont familiarisés avec les millions d'années pour les préhumains et les humains, les centaines de millions d'années pour les premiers reptiles et les premiers poissons, les milliards d'années pour les premiers êtres vivants et la dizaine de milliards d'années dépassée pour l'Univers, ses galaxies, certaines de ses étoiles, et bien sûr les planètes des étoiles en question. La dimension du temps s'est désormais bien installée dans les mentalités, du moins l'idée de son immensité car ses valeurs sont souvent énoncées à quelques zéros près. La première datation absolue $d^{\prime} 1750000$ ans d'un hominidé fossile à Olduvai en Tanzanie grâce à la mesure de la désintégration du potassium en argon dans un cristal d'un dépôt volcanique surmontant le niveau du crâne, avait fait grand bruit lors de sa publication qui ne remonte pourtant qu'à 40 ans. II s'en était suivi la vague habituelle et attendue de scepticisme et de rejet frileux. L'annonce des 3 millions
200000 ans de Lucy, celle dès 8 millions d'années d'origine de notre rameau, même accompagnées chaque fois de l'erreur de la mesure qui s'impose, ne posent plus de problèmes à personne.

Je terminerai volontiers ces quelques propos en vrac par une prospective pour I'humanité. La pensée de l'homme - la caractéristique du degré de complexité de sa matière - et l'acquisition des connaissances de son esprit, n'ont cessé de progresser depuis les 3 millions d'années de son existence et au travers des 100 milliards d'encéphales qui jusqu'ici les ont illustrées, et ce progrès s'est fait en particulier dans le sens de I'allongement du projet des initiatives de ce drôle de petit mammifère. C'est encore une question de mesures de durées. L'homme qui aménage un galet de silex il y a deux millions d'années le fait en effet pour se servir de l'objet qui en est résulté dans les minutes ou les heures qui suivent immédiatement la réalisation de cet outil. Celui qui taille un biface à double symétrie il y a 500000 ans le fait avec un tel soin dans le choix de la matière et dans la réalisation de l'objet et un tel souci d'efficacité et d'esthétique à la fois, qu'il a dû envisager de s'en servir des semaines, voire des mois. Celui qui recueille et qui perce dents et coquilles il y a 50000 ans pour réaliser un collier ou une chevillère pour offrir, a bien dû penser que son cadeau durerait des années. Celui qui, il y a 20000 ans, crée une couleur par mélange de quelques produits minéraux et végétaux recueillis à cette intention et qui prend soin d'y ajouter sable ou argile et sang de bison pour la "dégraisser », l'empêcher de craquer, la fixer et lui donner plus longue vie, a bien dûespérer que la peinture qu'il réalisait avec cette couleur durerait plusieurs générations et servirait à leurs initiations. L'astrophysicien qui, il y a 50 ans, informe ses contemporains que notre étoile, le soleil, n'a plus que 5 milliards d'années d'existence et que, par suite, si nous n'anticipons pas cet événement, nous nous condamnons nous-mêmes, n'a rien fait d'autre que de rallonger le temps de la prévision. C'est à ce point du temps universel que nous sommes parvenus. II nous faut donc maintenant songer au temps de la cellule et à celui de chacun - ce numéro s'en occupe avec sciences et élégance - mais aussi au temps de l'humanité; les solutions ne sont pour sa survie à terme pas nombreuses - déplacer les hommes ou déplacer la Terre - mais il n'y a pas de raison de penser que nous ne parviendrons pas à les mettre à exécution. Ce temps-là est certes à venir mais le temps d'avant et le temps d'après ont, que l'on sache, le même écoulement; ce temps-là pourrait donc bien ainsi absorber aussi le temps en biologie

\section{TIRÉS À PART}

Y. Coppens.

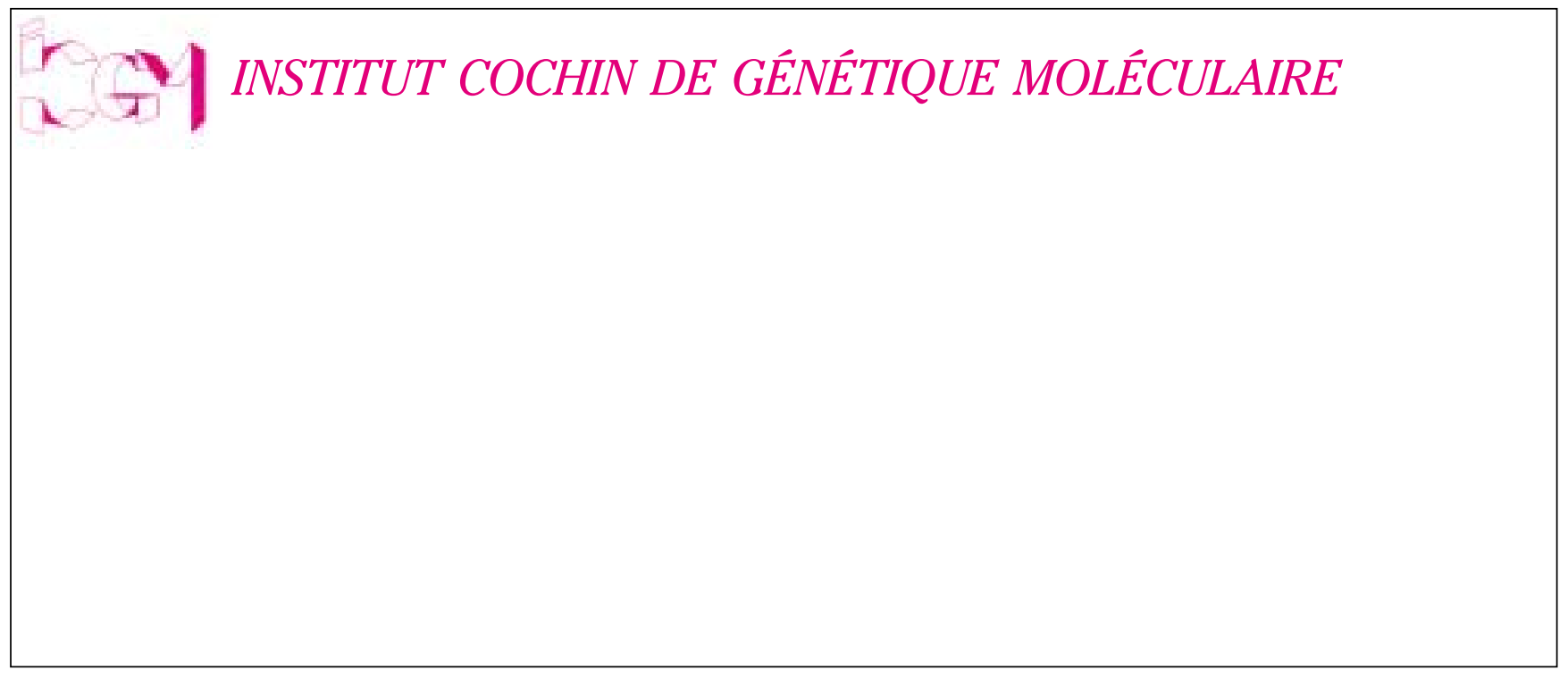

